\title{
Improving the sensitivity of immunoassays with PEG-COOH-like film prepared by plasma-based technique
}

\author{
Christy Charlton O'Mahony, ${ }^{1}$ Vladimir Gubala, ${ }^{1}$ Ram Prasad Gandhiraman, ${ }^{1}$ Stephen Daniels, ${ }^{1,2}$ \\ Jong Seol Yuk, ${ }^{1}$ Brian D. MacCraith, ${ }^{1}$ David E. Williams ${ }^{1,3}$ \\ ${ }^{1}$ Biomedical Diagnostics Institute, Dublin City University, Dublin 9, Ireland \\ ${ }^{2}$ National Centre for Plasma Science and Technology, Dublin City University, Dublin 9, Ireland \\ ${ }^{3}$ Department of Chemistry, MacDiarmid Institute for Advanced Materials and Nanotechnology, \\ University of Auckland, Auckland 1142, New Zealand
}

\begin{abstract}
Herein we report on a preparation and performance of stable, hydrophilic and biocompatible polymeric material suitable for functionalization of disposable substrates used in biosensors. This new material features $-\mathrm{COOH}$ surface groups cross-linked with ethylene glycol molecules and was prepared in situ on disposable, plastic substrate by high-throughput and environmentally friendly technique called plasma-enhanced chemical vapor deposition (PECVD). The film is grafted to the plasma activated plastic by sequential deposition of tetraethylorthosilicate, forming a bonding layer, and mixed vapors of acrylic acid and diethyleneglycol dimethylether (AA/PEG) that provide the desired functional groups forming a sensing, contact layer. A superior performance of the AA/PEG coating as suitable material for substrates in biomedical devices was demonstrated in a model
\end{abstract}

\section{INTRODUCTION}

In biomedical diagnostics, the chemical composition of the substrate surface and the subsequent cell or protein/surface interactions are considered to be the crucial factors defining the background response and effectively controlling the sensitivity of the device. In a successful bioassay device, it is absolutely critical to reduce the nonspecific binding (NSB) of the detection molecules in the absence of the target analyte, thus eliminate 'false positive' results. Therefore, surface coatings that can be mass-produced and combine the quality of low nonspecific binding and simultaneously have the ability to covalently immobilize biomaterial are of particular interest in biosensor applications. Poly- (ethylene glycol) (PEG) in different forms has proven to be a suitable material for construction of protein-resistant biosensor surfaces. $^{1-4}$ The low NSB function of PEG-molecules is associated with vast hydration and effects of change in configurational fluorescence linked immunosorbent assay. The results were compared with other commonly used surface materials prepared by wet chemistry methods. The unique characteristic of the AA/PEG film is that the immunoassay can be executed without the need for a blocking step, typically using albumins, without negative consequences on the bioassay results. In fact, the superior quality of the materials modified with $A A / P E G$ film was highlighted by improving the sensitivity of an immunoassay by two orders of magnitude when compared with substrates prepared by standard surface chemistry methods. (c) 2011 Wiley Periodicals, Inc. J Biomed Mater Res Part A: 100A: 230-235, 2012.

Key Words: surface chemistry, immunoassay, biosensor, plasma enhanced chemical vapor deposition 


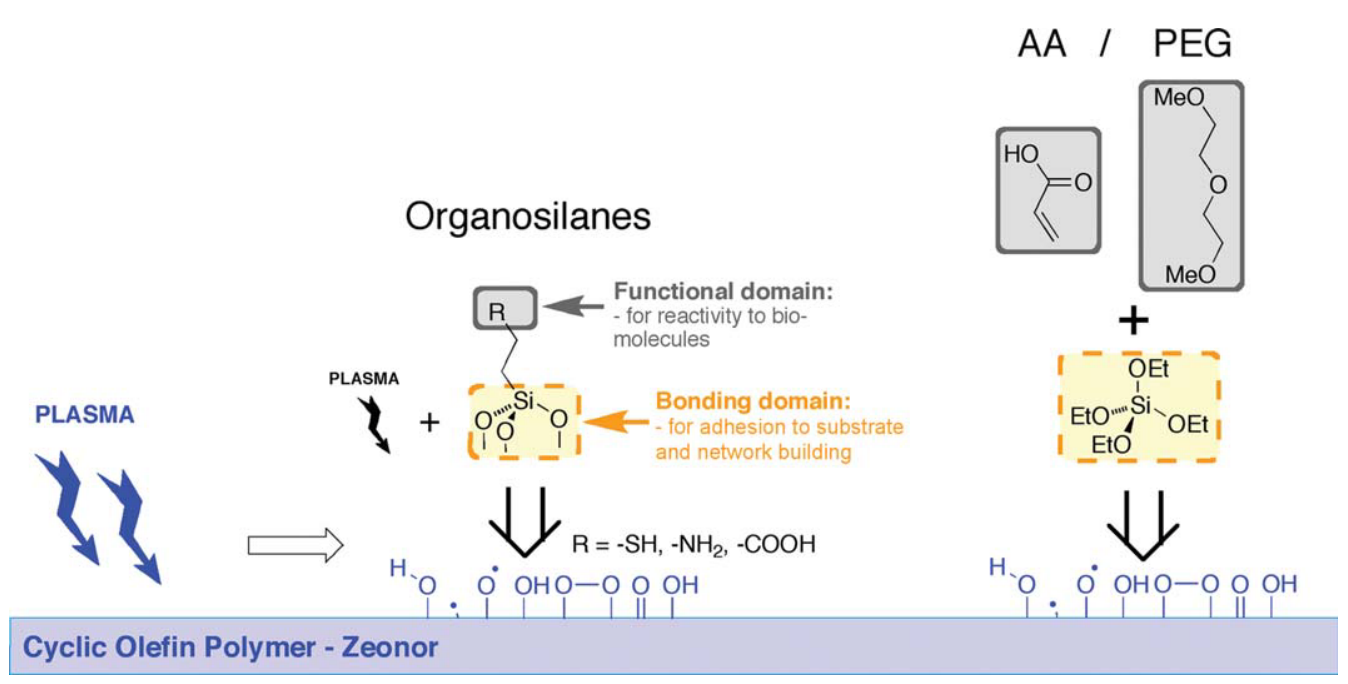

FIGURE 1. A schematic illustration of the in-situ PECVD process. COP substrates are oxidized in a plasma reactor and subsequently exposed to vapors of organosilanes that polymerize to form films with $-\mathrm{SH},-\mathrm{NH}_{2}$, or $-\mathrm{COOH}$ functionality. Alternatively, tetraorthosilicate is deposited to form the bonding layer, onto which mixture of precursors such as acrylic acid (AA) and diethylene glycol dimethylether (PEG) are grafted. [Color figure can be viewed in the online issue, which is available at wileyonlinelibrary.com.]

vapor pressure. Therefore, the development of alternative routes to functionalize plastic material for bioassay devices remains of great importance.

Here we present a generic method using tetraorthosilicate (TEOS) as a siloxane-containing precursor to form a bonding layer with the substrate, onto which acrylate (further in the text referred as AA film) or methacrylate monomers together with a short PEG derivative, diethylene glycol dimethyl ether (DEGDME) could be sequentially grafted (Fig. 1). The function of the PEG precursor is to act as a protein-resistant coreactant. ${ }^{1,3,5,13,16}$ Exposure of the plastic substrate to evaporating TEOS followed by vapors containing a mixture of molecules of DEGDME and acrylic acid produced a stable film containing both the reactive carboxylic acid groups and repellent PEG-like groups (further in the text referred as AA/PEG film). Once activated, the available - $\mathrm{COOH}$ groups could be used to covalently immobilize biorecognition elements like antibodies. The protein resistant PEG functionality on the other hand, should contribute to the reduction of the nonspecific binding of the nonanalyte constituents of the sample. The expected net effect is an improved signal-to-noise ratio. Therefore, the central objective of this communication is to demonstrate the quality of such surface with embedded - $\mathrm{COOH}-\mathrm{PEG}$-like functionality in a model sandwich immunoassay. The efficiency of the PECVD prepared coating to reduce NSB was compared with several other films, frequently used in bioassays. A human IgG, fluorescence linked immunosorbent assay was performed with selected surfaces to show how increase in signal/noise ratio directly translates into improvements in assay sensitivity.

\section{MATERIALS AND METHODS}

\section{Surface chemistry}

Wet chemistry methods. Plain COP slides (Zeonor ${ }^{\circledR}$ 1060R) $75 \mathrm{~mm} \times 25 \mathrm{~mm}$ were obtained from Sigolis AB (Uppsala, Sweden), $3 \times 3$ parabola element biochip was injection molded in Zeonex ${ }^{\circledR}$ copolymer (Nalux, Japan). All substrates were oxidized in oxygen plasma. The plasma oxidation of the material took place during $1 \mathrm{~min}$ at $200 \mathrm{mTorr}, 200 \mathrm{~W}$ and with a flow of oxygen at $100 \mathrm{~mL} / \mathrm{min}$, in expanded plasma cleaner (PDC- 002, Harrick Science, Ossing, NY).

PDITC coating. After plasma oxidation, the $3 \times 3$ parabola element biochip as well as $2.5 \times 7.5 \mathrm{~cm}^{2}$ COP slides were immersed in a solution of $3 \mathrm{vol} \% 3$-Aminopropyltriethoxysilane (APTES, Fluka) in $95 \%$ ethanol for $2 \mathrm{~h}$, similar to the process used for glass surfaces. ${ }^{5}$

For reaction with PDITC, the APTES-modified COP substrates were immersed in a $25 \mathrm{mM}$ PDITC in DMF:pyridine $(9: 1, v / v)$ solution for $2 \mathrm{~h}$. The slides were then rinsed with $\mathrm{DMF}$ and $\mathrm{MeOH}$ and dried under a stream of nitrogen.

Oxidized dextran coating. APTES coated chips were immersed in an oxidized 2\% dextran solution (Dextran T40 (40 kDa), Pharmacosmos, Denmark) for $2 \mathrm{~h}^{1}$ rinsed in MilliQ- $\mathrm{H}_{2} \mathrm{O}$ and further oxidized in $30 \mathrm{mM} \mathrm{NaIO}$ (Sigma Aldrich) for $2 \mathrm{~h}$.

PECVD method. The deposition of $-\mathrm{COOH}$ and $-\mathrm{COOH} /$ DEGDME functional coatings was carried out in a computer controlled PECVD reactor Europlasma, model CD300 (Oudenaarde, Ghent, Belgium). An aluminum vacuum chamber, connected to a Dressler CESAR 136 RF power source (Munsterau, Stolberg, Germany) with an operating frequency of 13.56 MHz, with an automated impedance-matching box, was used. The details of the deposition system is provided elsewhere. ${ }^{17}$ TEOS, AA, and diethylene glycol dimethyl ether were contained in three separate single side blocked KF16 nipples (Kurt J Lesker, UK) connected to the chamber individually through needle valves. The needle valves were used to control the sequential flow of vapors of tetraethylorthosilicate (TEOS) and acrylic acid (AA) for AA coating and a mixture of vapors of acrylic acid and diethylene glycol 

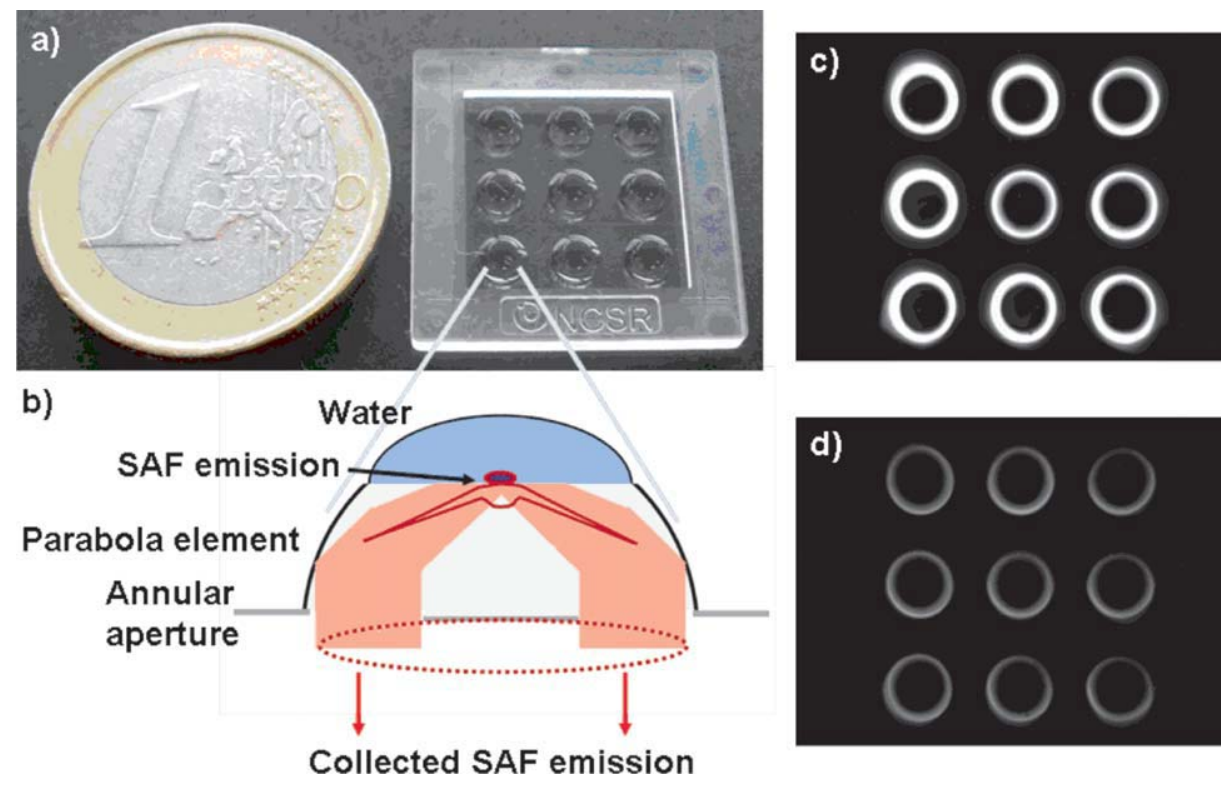

FIGURE 2. The parabolic chip used is shown (a) pictorially and (b) schematically. Collected images of background levels from an unwashed chip coated with (c) PDITC and (d) AA/PEG are shown with the same gain setting. [Color figure can be viewed in the online issue, which is available at wileyonlinelibrary.com.]

dimethyl ether for AA/PEG film. Plasma pretreatment was carried out at an RF power of 250 Watts for 3 min with $50 \mathrm{sccm}$ of argon flow and $50 \mathrm{sccm}$ of oxygen flow. The plasma deposition was carried out in argon plasma. Plasma pretreatment, deposition of TEOS and AA/PEG were all carried out sequentially.

\section{Fluorescence linked immunosorbent assay}

Human IgG from serum, goat anti-human IgG (Fab specific) and bovine serum albumin (BSA) were purchased from Sigma (St. Louis, MO). Alexa Fluor 647 labeled goat antihuman IgG was purchased from Molecular Probes ${ }^{\text {TM }}$ (Eugene, OR). Capture antibody (goat anti-human IgG) was immobilized on each surface by incubation of a solution of $400 \mu \mathrm{g} / \mathrm{mL}$ in Phosphate buffered saline (PBS) containing $1 \% \mathrm{v} / \mathrm{v}$ Tween 20 on the surface in a humid chamber at $37^{\circ} \mathrm{C}$ for $1 \mathrm{~h}$. For covalent immobilization of antibodies on the AA and AA/PEG films, the - $\mathrm{COOH}$ surface groups were activated by immersing the substrates into $100 \mathrm{mM}$ aqueous solution of 1-Ethyl-3-[3-dimethylaminopropyl]carbodiimide Hydrochloride (EDC) and $100 \mathrm{mM}$ of $N$-hydroxysiccinimide (NHS) for $10 \mathrm{~min}$. The slides were then rinsed one time with MilliQ water and 1-3 $\mu \mathrm{L}$ droplets of goat anti-human IgG at a concentration of $400 \mu \mathrm{g} / \mathrm{mL}$ were transferred on to the activated surface and incubated at $37^{\circ} \mathrm{C}$ for $1 \mathrm{~h}$. The slides were then rinsed with a PBS solution containing Tween $20(1 \%, v / v)$ for $10 \mathrm{~min}$, followed by 5 -min rinse with MilliQ water and dried with a stream of nitrogen. For all measured surfaces, except for AA/PEG this was followed by a blocking step of incubation of a solution of $1 \% \mathrm{w} / \mathrm{v}$ BSA in PBS Tween for $1 \mathrm{~h}$ at room temperature. For assay measurements, IgG sample solutions were prepared by diluting IgG in PBS Tween solution. Eight concentrations were prepared by diluting in steps of ten $(100,000,10,000$,
1000, 100, 10, 1, 0.1, $0.01 \mathrm{ng} / \mathrm{mL}$ ). Plain PBS buffer was used as a negative control and also to assess the level of nonspecific binding $(\sim 0 \mathrm{ng} / \mathrm{mL})$. Chips and slides were washed for $10 \mathrm{~min}$ in PBS Tween followed by $5 \mathrm{~min}$ in DI water and were blown dry with nitrogen. Finally, labeled antibody (Alexa fluor 648-labeled goat anti-human IgG) was added at a concentration of $200 \mu \mathrm{g} / \mathrm{mL}$ in PBS Tween and was incubated for $1 \mathrm{~h}$ at $37^{\circ} \mathrm{C}$. In all cases, a volume of $1 \mu \mathrm{L}$ was used for the $3 \times 3$ parabola element biochip and a volume of $3 \mu \mathrm{L}$ was used for the microscope size slides. The fluorescence signal of the COP slides was recorded on PerkinElmer ScanArray Express (PerkinElmer, MA) with laser excitation wavelength of $633 \mathrm{~nm}$ and emission filter wavelength of $670 \mathrm{~nm}$. The fluorescence signal of the $3 \times 3$ parabola element biochips was measured as described below.

\section{Supercritical angle fluorescence measurement}

The $3 \times 3$ parabola element biochip is shown pictorially and schematically in Figure 2. The chip is designed to be illuminated from below in an optics system discussed previously. ${ }^{7}$ This system allows for total internal reflection fluorescence (TIRF) excitation and supercritical angle fluorescence (SAF) detection giving it a high surface specificity. Briefly, a laser beam is expanded to illuminate all nine parabolas simultaneously. Light couples to the top surface of the parabola and fluorophores at the surface are excited via the evanescent wave. These surface-bound fluorophores then emit much of their light at high angles (larger than the critical angle) causing it to typically be trapped inside the substrate. The shape of the parabola element is designed to recover this light as shown schematically in Figure 2. An annular aperture is used to ensure that only light from surface-bound fluorophores is collected and imaged by the CCD 
TABLE I. Water Contact Angles and Thickness of Coatings Prepared by Wet Chemistry Methods and PECVD

\begin{tabular}{clrc}
\hline & & $\begin{array}{c}\text { Water Contact } \\
\text { Angle }\end{array}$ & $\begin{array}{c}\text { Thickness } \\
{[\mathrm{nm}]}\end{array}$ \\
\hline \multirow{4}{*}{ Wet chemistry } & Pristine COP & $92.35^{\circ} \pm 1.55^{\circ}$ & - \\
methods & APTES & $55.05^{\circ} \pm 2.60^{\circ}$ & $13.39 \pm 0.48$ \\
& PDITC & $70.43^{\circ} \pm 1.26^{\circ}$ & $15.21 \pm 0.22$ \\
PECVD & Oxid. dextran & $30.19^{\circ} \pm 0.28^{\circ}$ & $15.23 \pm 0.10$ \\
& AA & $9.85^{\circ} \pm 0.21^{\circ}$ & $5.70 \pm 1.40$ \\
& AA/PEG & $18.41^{\circ} \pm 0.96^{\circ}$ & $5.59 \pm 0.70$ \\
\hline
\end{tabular}

camera producing the images shown in Figure 2(c,d). Fluorescence intensities were calculated by measuring the sum of the light in the ring created by each parabolic SAF collection element using Matlab image processing.

\section{RESULTS AND DISCUSSION}

\section{Surface chemistry}

The functionalization of plastic substrates, such as COP, for bioassay applications has recently attracted lots of scientific and technological interest. Surface functionalization of COP carried out by wet chemistry methods is relatively well known. To our best knowledge, the two methods we have chosen to coat the plastic surface are currently being explored for the use in commercial bioassay devices. ${ }^{18}$ Material modified with 1,4-phenyldiisothiocyanate (PDITC) surface is also commercially available. ${ }^{6}$ The surface modification by wet chemistry requires a two-step process, in which large amount of solvent is used. The first step
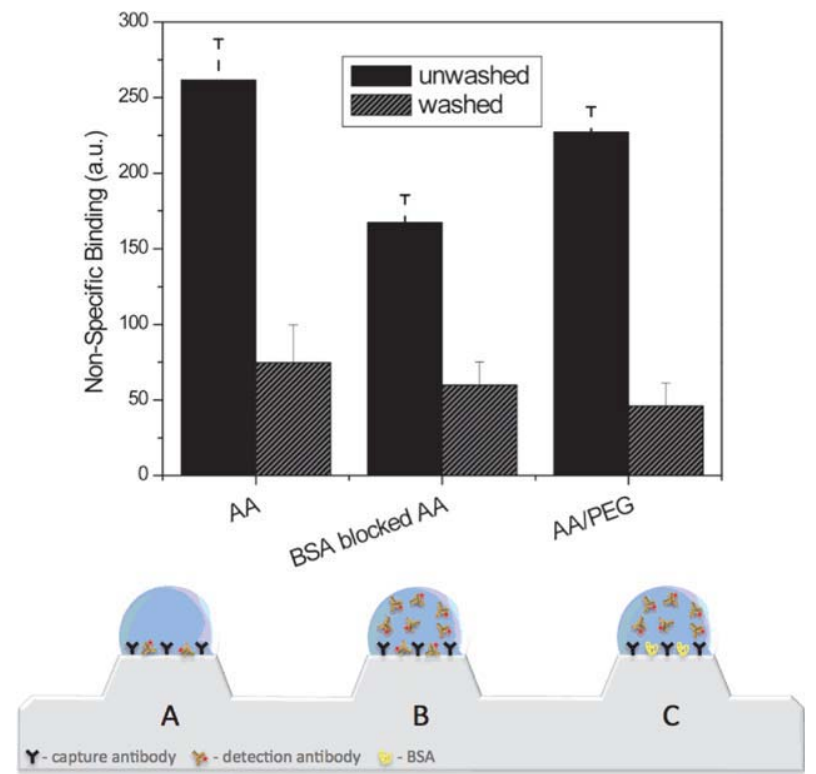

FIGURE 3. Top-The fluorescence signal of the labeled, detection antibodies, presented as nonspecific binding was measured at zero concentration of the analyte. The degree of binding of the detection molecules to the surface in the absence of the analyte controls the background response and hence the sensitivity of an assay. Bottoma cartoon representation illustrating the design of the experiments on washed (a), unwashed (b), and unwashed-BSA blocked (c) $3 \times 3$ parabola element biochips. [Color figure can be viewed in the online issue, which is available at wileyonlinelibrary.com.] involves silanization of the plastic substrate, using (3-aminopropyl)dimethylethoxysilane (APTES), to create an adhesive layer on oxidized COP. The resultant amino terminated surface is subsequently functionalized with either a dextran matrix or PDITC cross-linker, which allows for immobilization of capture antibodies. On the other hand, the PECVD process represents a straightforward, industrially scalable, one step, in-situ and solvent-free method. As seen in Table I, treatment of the COP by PECVD techniques as well as by oxidized dextran (oxid. dextran) renders the surface hydrophilic, which is in contrast to the PDITC film. The film thicknesses of various films are also given in Table I. The apparent higher error variations in the thickness of the PECVD prepared surfaces are attributed to a very specific deposition approach, as illustrated on Figure 1. The reaction mechanism involves an activation of the substrate with high-energy plasma, followed by deposition of partially fragmented precursor vapors under low-energy plasma. However, it is necessary put this into a perspective with the size of the antibodies that are immobilized on such surface. If the antibody footprint is considered to be $100 \mathrm{~nm}^{2}$, the error values of 1.4 or $0.7 \mathrm{~nm}$ for the PECVD films are considered almost negligible. Overall, we reason that the capacity of AA/PEG film to reduce nonspecific adsorption of proteins is due to a proper combination of various factors such as the changes in surface potential polarity, adhesion force, effects of swelling in buffered solution and "hardness" of the surface.

\section{Nonspecific binding measurements on supercritical angle fluorescence system}

To compare the effect of nonspecific adsorption on the prepared surfaces, we used an in-house designed detection

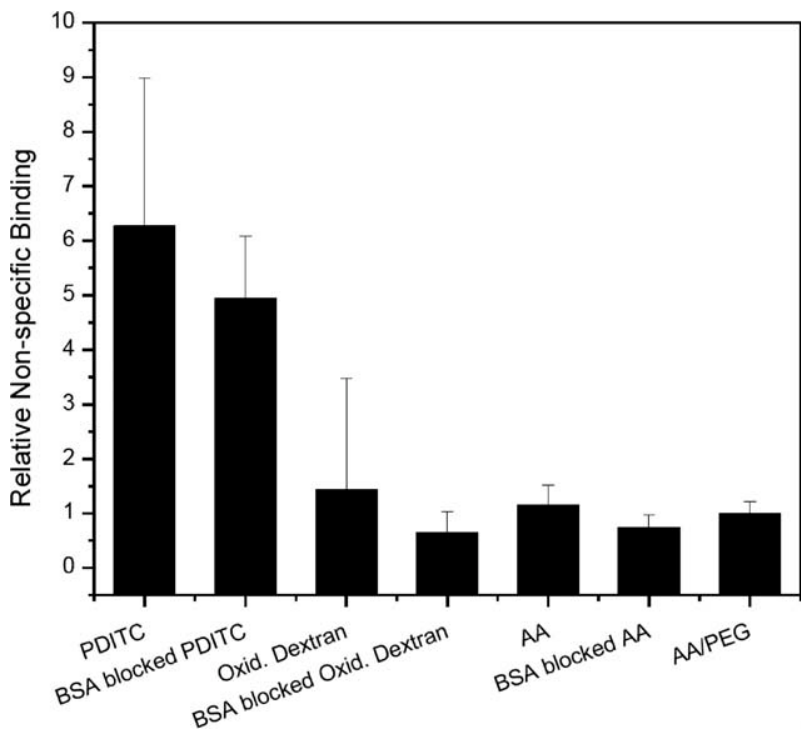

FIGURE 4. Relative nonspecific binding, presented as an increment of fluorescence signal change of studied coatings relative to fluorescence signal of AA/PEG surface. The fluorescence of labeled detection antibodies was measured on unwashed $3 \times 3$ parabola element biochips at zero concentration of analyte. The experiment design is also illustrated in Figure 3(A). 

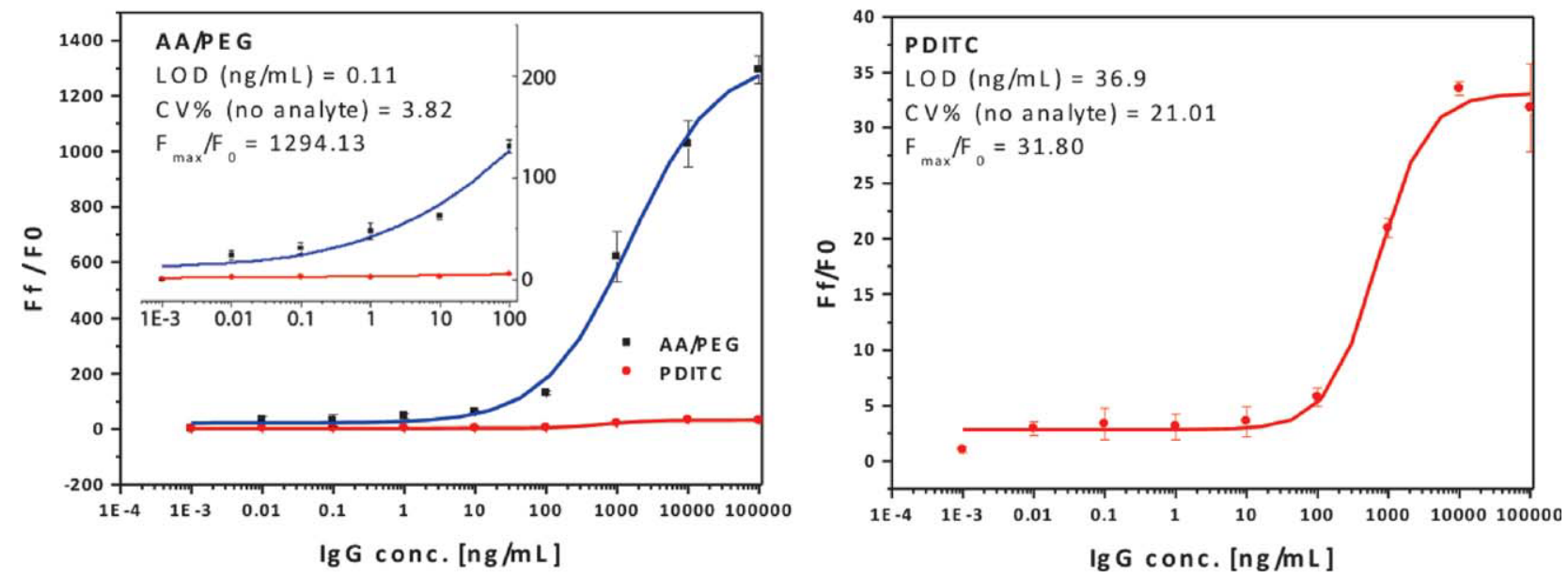

FIGURE 5. Human IgG immunoassay curves for unblocked AA/PEG (blue curve) modified surface and BSA blocked PDITC (red curve) modified COP slides. Inset shows the region of the calculated limit of detection for both surfaces. [Color figure can be viewed in the online issue, which is available at wileyonlinelibrary.com.]

system based on Supercritical Angle Fluorescence (SAF) ${ }^{7}$ The principle is illustrated in Figure 2. The biochip is made of injection molded COP with nine parabolic elements that allow for surface specific fluorescence collection. The discrimination of the bulk fluorescence prompted us to investigate the ability to measure "unwashed" samples, as illustrated in Figure 3(B). Under this experimental setup, the fluorescently labeled detection molecules would be specifically bound to the analytes in the typical sandwich format, with large excess of unbound labeled molecules in the bulk solution. Because it is desirable to prevent binding of the detection molecules to the surface in the absence of the target analyte (in order to eliminate "false positive" results), it is a good practice to block the surface after the immobilization of capture antibodies with blocking reagent, such as BSA. While the fluorescence intensities from AA, BSA blocked AA, and AA/PEG coated chips were already reasonably low, washing the excess of the bulk detection antibody further decreased the background fluorescence signal of up to four times. The effect of BSA to prevent nonspecific binding of proteins is evident in both Figures 3 and 4 . Figure 4 shows an increment of fluorescence signal change measured on unwashed chips functionalized with wet chemistry methods relative to that of AA/PEG. In this light, the AA/PEG surface compared favorably with all other surfaces. Only biochips with BSA blocked AA and oxidized dextran films showed lower background response than AA/PEG chemistry (although with no BSA blocking step). The oxidized dextran coating also showed reduction in binding levels when blocked with BSA, which is attributed to the polar nature of the polysaccharide and high degree of hydration. However, in the absence of BSA, it also showed relatively high variations between the spots and poor uniformity.

\section{Fluorescence linked immunosorbent assay}

The immunoassay was carried out by immobilization of goat-anti-human IgG on both the PDITC and AA/PEG modified COP slides. The analyte, human IgG, was then allowed to react with the surface captured antibody and the amount of analyte was detected by reaction with Alexa 648-goat-anti-human IgG antibody. The concentrations of the capture and detection antibodies were optimized to 0.4 and $0.2 \mathrm{mg} / \mathrm{mL}$, respectively. The PDITC surface was blocked with $1 \% \mathrm{w} / \mathrm{v}$ solution of BSA after the immobilization of captured antibody, while the AA/PEG surface was used without the extra blocking step. These optimal values were then used for the immunoassay. The limit of detection (LOD) for PDITC surface was calculated to be $36.9 \mathrm{ng} / \mathrm{mL}$. The value for the AA/PEG surface was determined to be $0.11 \mathrm{ng} / \mathrm{mL}$, showing an improvement by two orders of magnitude. The LOD was calculated as concentration that corresponds to the background signal plus three times the standard deviation of the background signal. Theses values are of similar order to the LODs obtained for immunoassays on other PDITC coated COP, silicon or glass substrates. ${ }^{8-11}$ Because of significantly lower background signal on the AA/PEG slide, not even blocked by BSA, the LOD improved by two orders of magnitude and the maximum fluorescence $F_{\text {max }} / F_{0}$ was more than 35 times better on the PECVD coated substrate than the one prepared by standard PDITC surface chemistry. This effectively demonstrates the superior properties of AA/PEG coating in a model sandwich immunoassay.

\section{CONCLUSION}

In this work, carboxylic-functionalized film in a matrix of protein-resistant PEG was fabricated on cyclo olefin polymer. Such a film with its highly reactive carboxy sites enabled covalent attachment of biomolecules on the surface, while its low reactivity PEG sites prevented the nonspecific binding of other nonanalyte solution constituents. The surface capacity of suppressing background fluorescence was demonstrated on a prototype of biochip device based on supercritical angle fluorescence detection. A model immunosorbent assay was performed to illustrate how 
reduction in background response translates into improvements in signal-to-noise ratio and subsequently to improved limit of detection. The sensitivity improved by two orders of magnitude as a direct consequence of the optimal surface chemistry. The effect of the carboxy coating with incorporated PEG matrix was compared with other films prepared by either wet chemistry methods or plasma-assisted vapor technique. The results suggest that the PECVD process of codepositing reactive carboxylic functionality and repellant PEG functionality is indeed capable of preparing surfaces for mass industrial production, characteristics of which are comparable or even better to those that are frequently used in immunoassays by other scientific and industrial entities.

\section{ACKNOWLEDGMENTS}

The authors wish to thank Ruairi Monaghan and Taiwo Oduneye for their help with thickness and contact angle measurements and Conor Coyle for help with PECVD surface preparation.

\section{REFERENCES}

1. Valsesia A, Colpo $P$, Meziani $T$, Bretagnol $F$, Lejeune $M$, Rossi $F$ Bouma A, Garcia-Parajo M. Adv Func Mater 2006;16:1242-1246.

2. Miyachi H, Ikebukuro K, Yano K, Aburatani H, Karube I. Biosens Bioelectron 2004;20:184-189.

3. Chu LQ, Knoll W, Forch R. Plasma process. Polymer 2006;3(6-7): 498-505.

4. Miyachi H, Hiratsuka A, Ikebukuro K, Yano K, Muguruma H, Karube I. Biotechnol Bioeng 2000;69:323-329.

5. Sardella E, Gristina R, Senesi GS, d'Agostino R, Favia P. Plasma Process Polymer 2004;1:63-72.

6. Available at: http://www.asperbio.com/ASPER_COMPANY.PDF. ASPER BIOTECH Ltd.

7. Yuk JS, Trnavsky M, McDonagh C, MacCraith BD. Surface plasmon-coupled emission (SPCE)-based immunoassay using a novel paraboloid array biochip. Biosens Bioelectron 2010;25.
8. Boozer C, Ladd J, Chen S, Jiang S. DNA directed protein immobilization on mixed ssDNA/Oligo(ethylene glycol) self-assembled monolayers for sensitive biosensors. Anal Chem 2006;78: 1515-1519.

9. Boozer C, Ladd J, Chen S, Yu Q, Homola J, Jiang S. DNA-directed protein immobilization for simultaneous detection of multiple analytes by surface plasmon resonance biosensor. Anal Chem 2004;74:6967-6972.

10. Vilholm-Lundin I, Albers W. Site-directed immobilisation of antibody fragments for detection of C-reactive protein. Biosens Bioelectron 2006;21:1141-1148.

11. Raj J, Herzog G, Manning M, Volcke C, MacCraith B, Ballantyne S, Thompson M, Arrigan DWM. Surface immobilisation of antibody on cyclic olefin copolymer for sandwich immunoassay. Biosens Bioelectron 2009;24:2654-2658.

12. Evans CW, Gubala V, Nooney R, Williams DE, Brimble MA, Devries AL. How do antarctic notothenioid fishes cope with internal ice? A novel function for antifreeze glycoproteins. Antarct Sci 2010;23:57-64.

13. Larsson A, Ekblad T, Andersson O, Liedberg B. Photografted poly(ethylene glycol) matrix for affinity interaction studies. Biomacromolecules 2007;8:287-295.

14. Gandhiraman RP, Gubala V, Le CHN, Volcke C, Doyle C, James B, Daniels S, Williams DE. Deposition of chemically reactive and repellent sites on biosensor chips for reduced non-specific binding. Colloids Surface B 2010;79:270-275.

15. Gubala V, Gandhiraman RP, Volcke C, Doyle C, Coyle C, James B, Daniels S, Williams DE. Silanation of cyclo olefin polymer surfaces. Comprehensive characterization of (3-Aminopropyl) trisethoxysilane coatings by PECVD: from the Contact Layer into the Bulk. Analyst 2010;135:1375-1381.

16. Silván MM, Valsesia A, Hasiwa M, Gilliland D, Ceccone G, Rossi F. Surface characterization of biopolymer micropatterns processed by ion-beam modification and PECVD. Chem Vapor Depos 2007;13:211-218.

17. Gandhiraman RP, Karkari SK, Daniels S, MacCraith B. Influence of ion bombardment on the surface functionalization of plasma deposited coatings. Surf Coat Technol 2009;203: 3521-3526.

18. Jonsson C, Aronsson M, Rundstrom G, Pettersson C, MendelHartvig I, Bakker J, Martinsson E, Liedberg B, MacCraith B, Ohman O. Lab Chip 2008;8:1191-1197. 